12

\title{
Влияние дрейфа частоты лазера в фазочувствительной рефлектометрии
}

\author{
(C) А.А. Жирнов ${ }^{1}$, К.В. Степанов ${ }^{1}$, А.О. Чернуцкий ${ }^{1}$, А.К. Федоров ${ }^{2}$, Е.Т. Нестеров ${ }^{1}$, \\ Ч. Звелто ${ }^{3}$, А.Б. Пнев ${ }^{1}$, В.Е. Карасик ${ }^{1}$ \\ ${ }^{1}$ МГТУ им. Н.Э. Баумана, 105005 Москва, Россия \\ ${ }^{2}$ Российский квантовый центр, 143025 Сколково, Москва \\ ${ }^{3}$ Dipartimento di Elettronica e Informazione del Politecnico di Milano, 20133 Milano, Italy \\ e-mail: a.zh@bmstu.ru
}

Поступила в редакцию 11.06.2019 г.

В окончательной редакции 11.06.2019 г.

Принята к публикации 20.06.2019 г.

Рассмотрено влияние флуктуаций частоты лазера на работу фазочувствительных рефлектометрических (Ph-OTDR) систем. Сообщено о теоретических результатах на основании новой числовой модели Ph-OTDR, демонстрирующей влияние нестабильности частоты лазера на сигнал. Модель была верифицирована на основе экспериментальных данных. На ее основе было рассчитано отношение сигнал/шум (SNR) системы для различных параметров стабильности лазерного источника. Это позволило сформулировать количественные требования к лазерам для систем Ph-OTDR.

Ключевые слова: фазочувствительный рефлектометр, флуктуация частоты лазера, узкополосный лазер, распределенный датчик.

DOI: $10.21883 /$ OS.2019.10.48364.177-19

\section{Введение}

Распределенные датчики вибрации, основанные на методе фазочувствительной рефлектометрии, весьма перспективны для удаленного контроля протяженных объектов, таких как мосты, дороги, трубопроводы и др. [1]. $\mathrm{B}$ отличие от обычных датчиков OTDR узкополосный зондирующий сигнал позволяет обнаруживать возмущения по их влиянию на сигналы (рис. 1), которые формируются с учетом фаз обратно рассеянных волн [2-4]. В последние десятилетия сенсорные системы на основе Ph-OTDR вызывают значительный интерес [2-13]. Последние разработки в производстве волоконно-оптических компонентов приводят к значительному снижению себестоимости таких сенсорных систем и повышению качества их работы, что увеличивает число потенциальных областей применения.

Принцип работы системы можно описать на примере схемы на рис. 1 следующим образом. Излучение узкополосного источника 1 доводится до необходимого уровня мощности в бустере 2, формируется в импульсы на акустооптическом модуляторе 3. Далее через циркулятор 4 направляется в сенсорное волокно 5 . Обратно рассеянное излучение сначала усиливается в предусилителе 6. Спонтанное излучение отсеивается оптическим фильтром 7. Затем сигнал регистрируется фотоприемным устройством 8 , оцифровывается АЦП 9 и обрабатывается на ПК 10. Сигнал в данной системе проявляется, когда на каком-то участке сигнал начинает резко флуктуировать относительно своего уровня за последний промежуток времени.
Фазочувствительная рефлектометрия реализуется только в том случае, если длина когерентности источника излучения не меньше длительности импульса [2-4]. Таким образом, лазер является определяющим компонентом системы. В целом, качество сенсорных систем для удаленного контроля протяженных объектов определяется их способностью обнаруживать возмущения, вызванные активностью вблизи волокна на фоне, формируемом как внешними шумами (например, сейсмическими), так и собственными шумами системы. Для изучения их влияния датчики на основе Ph-OTDR исследовались также на базе численного моделирования [10,14-20].

В некоторых исследованиях $[8,19]$ изучалась роль именно лазерного источника. В частности, было продемонстрировано, что частотный дрейф источников может уменьшать SNR и даже может вызывать ложные срабатывания. Однако в настоящее время не предложено количественных критериев стабильности частоты лазерных источников для датчиков на основе Ph-OTDR. Одной из причин является наличие различных типов лазеров для Ph-OTDR, обладающих особенностями. Исполь-

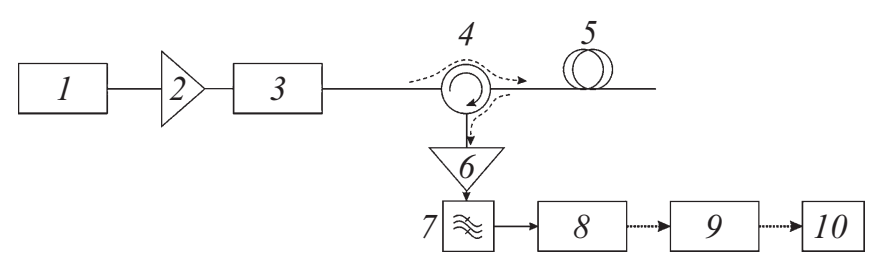

Рис. 1. Схема фазочувствительного рефлектометра. 
зуемые волоконные, твердотельные или полупроводниковые лазеры могут иметь флуктуации на различных частотах. Спектральная плотность мощности частотного шума может играть роль более общей характеристики. В настоящей работе будет предложено описание общего вида этой характеристики, что позволит в первом приближении компенсировать проблему ее различной формы для разных источников.

В настоящей работе изучается влияние нестабильности частоты лазерных источников на сигнал систем на основе Ph-OTDR с использованием численного моделирования и экспериментальных исследований. Прежде всего мы предлагаем новую численную модель, учитывающую флуктуации частоты во времени. Для ее описания мы приводим общую параметризованную форму спектральной плотности мощности флуктуаций частоты в качестве верхнего предела для текущей модели. Это предположение проверено и подтверждено экспериментальными данными трех разных лазеров. Далее, на основе сгенерированных массивов сигналов $\Phi-\mathrm{OTDR}$ c различными источниками, заданными параметрами стабильности частоты, рассчитаем SNR в аналогичных условиях для каждого лазера. Это позволит выбрать область параметров, которые удовлетворяют качественной работе сенсорной системы. Таким образом, требования к флуктуации частоты для источников в системах на основе Ph-OTDR, которые направлены на максимизацию верного определения событий, могут быть сформулированы численно.

\section{Численное моделирование}

Сигнал фазочувствительного рефлектометра складывается из обратно рассеянных волн со случайным распределением фаз. Такая природа обусловливает возможность только статистического описания данной системы. Для создания модели необходимо описать параметры ее элементарной частицы - одного центра рассеяния. Для составления модели используется тот факт, что рассеяние от всех центров является случайным комплексным сигналом [21]:

$$
\begin{gathered}
p_{A}(a)=\left\{\begin{array}{l}
\frac{a}{\sigma^{2}} \exp \left\{-\frac{a^{2}}{2 \sigma^{2}}\right\} \text { if } a>0, \\
0 \text { otherwise, }
\end{array}\right. \\
p_{\Theta}(\Theta)= \begin{cases}\frac{1}{2 \pi} & \text { if }-\pi<\Theta \leq \pi, \\
0 & \text { otherwise, }\end{cases}
\end{gathered}
$$

где $p_{A}(a)-$ плотность распределения амплитуды рассеяния, $p_{\Theta}(\Theta)$ - плотность распределения фазы рассеянных волн.

Необходимо учитывать, что амплитуда рассеяния также будет зависеть от расстояния до $n$-го центра рассеяния $L_{n}$ : необходимо учесть затухание $\exp \left(2 \alpha L_{n}\right)$, где
$\alpha[\mathrm{dB} / \mathrm{km}]$ - затухание в волокне, 2 - множитель, учитывающий прохождение света до центра рассеяния и обратно. Размер неоднородностей, на которых происходит релеевское рассеяние, составляет порядка 1/10 длины волны; соответственно для прибора, работающего на $\lambda \approx 1550 \mathrm{~nm}$, эта величина будет составлять примерно $d_{c}=150 \mathrm{~nm}$. Распределение в поперечном сечении моды можно не учитывать, так как фаза всех обратно рассеянных волн от этих центров будет одинакова. Соответственно для полного моделирования $L_{c}=50 \mathrm{~km}$ сенсора ориентировочно необходимо рассчитать число элементов

$$
N_{p} \approx \frac{L_{c}}{d_{c}}=\frac{50 \cdot 10^{3}}{150 \cdot 10^{-9}} \approx 3 \cdot 10^{11} \text { elements. }
$$

Так как мощности вычислительных компьютеров ограничены, то найдем способы сокращения объектов для модельного расчета. Так как коэффициент затухания $\alpha$ для рабочей длины волны $1550 \mathrm{~nm}$ в современных типах волокон составляет $\sim 0.2 \mathrm{~dB} / \mathrm{km}$, то обусловленные им различия между центрами рассеяния в пределах одного импульса (до $100 \mathrm{~m}$ ) можно считать пренебрежимо малыми. Кроме того, заметим, что нет необходимости моделировать весь сенсор целиком, так как событие обычно локализовано на участке не более $100 \mathrm{~m}$, так что задать длину сенсора $L_{c e}=500 \mathrm{~m}$, обеспечив ему соответствующее для исследуемого участка затухание, будет достаточно. Также допустим предварительное сложение волн с участка меньшего, чем пространственное разрешение прибора и длина волны в грунте. Распределение амплитуд рассеяния с этих участков $d_{e}$ также будет иметь форму релеевского распределения

$$
N_{p}=\frac{L_{c e}}{d_{e}}=\frac{500}{0.05}=10^{4} \text { elements. }
$$

Данная величина массива позволяет проводить моделирование без ухудшения качества результатов. При численном эксперименте необходимо будет помнить, что интерес представляет эволюция во времени рассеянного сигнала, т.е. необходимо будет сохранить $10^{4}$ элементов на протяжении как минимум $60 \cdot 10^{3}$ шагов, что будет эквивалентно одной минуте сигнала фазочувствительного рефлектометра, записанного с частотой $1 \mathrm{kHz}$.

Для получения информации о сигнале необходимо задать следующие основные параметры системы:

1. длину волны $\lambda_{t}$ или частоту $v_{t}$ лазерного источника для каждого момента запуска зондирующего импульса в сенсор;

2. амплитуду $A_{n} n$-го центра рассеяния, она является постоянной в течение всех эволюций модели;

3. вместо задания фазы каждого центра $\varphi_{n, t}$ необходимо задать расстояние от лазерного источника (или любой другой условной точки начала сенсора) до этого центра $L_{n}$; фаза будет рассчитываться по формуле

$$
\varphi_{n, t}=\frac{2 \pi}{\lambda_{t}} L_{n}=\frac{2 \pi n_{g} v_{t}}{c} L_{n}
$$


где $n_{g}$ - групповой эффективный показатель преломления сердцевины волокна.

Простым и эффективным вариантом будет задание равномерного увеличения удаления центров рассеяния от начала.

Набор вышеперечисленных параметров позволит получить значение комплексного сигнала от каждого задаваемого участка рассеяния:

$$
s_{t, n}=A_{n} e^{j \varphi_{n, t}}
$$

Далее необходимо получить сигнал, который регистрирует АЦП в каждый момент времени при регистрации одной рефлектограммы. Это квадрат модуля суммы с учетом фаз сигналов от центров рассеяния в половине длины оптического импульса:

$$
S_{t, n_{i}}=\left|\sum_{n=n_{i}}^{n_{i}+n_{i m p / 2}} s_{t, n}\right|^{2}
$$

где $n_{i}$ - первый центр рассеяния на текущем отсчете АЦП, $n_{i m p / 2}$ - число центров рассеяния, помещающихся на половину длины зондирующего импульса.

В модели сдвиг зондирующего импульса будет обозначаться через число элементов

$$
\Delta n_{s}=\frac{t_{A D C} c}{2 n_{e} d_{e}}
$$

В таком случае одну рефлектограмму из $K$ отсчетов на АЦП $\left(K=\frac{L_{c e}}{d_{3} \Delta n_{\text {д }}}\right)$ можно получить из следующего выражения:

$$
R_{\text {ind }, t}=\left.S_{t, n_{i}}\right|_{i=(\text { ind }-1) \Delta n_{\text {म }}+1} ^{K \Delta n_{s}+1},
$$

где ind - номер отсчета рефлектограммы.

Аналогично через специальные функции

$$
R_{i n d, t}=\operatorname{comb}\left(\frac{i}{\frac{t_{A D C} c}{2 n_{3} d_{3}}}\right)\left(\left.S_{t, n_{i}}\right|_{i=1} ^{\frac{L_{c e}}{d_{e}}} \otimes \operatorname{rect}\left(\frac{i}{\frac{\tau_{i m p} c}{2 n_{e} d_{e}}}\right)\right) .
$$

Данная формула полезна тем, что может быстрее задаваться для расчетов, а также позволяет моделировать отклонения формы импульса от идеальной при замене rect на численно заданную геометрию.

Для окончательного формирования одной рефлектограммы в виде, котором она будет отображаться на экране, необходимо учесть полосу пропускания электрического усилителя фотодиодного приемника. Так как самый высокочастотный сигнал (до $100 \mathrm{MHz}$ ) в системе содержит большую долю шумов, то обычно его отфильтровывают до диапазона от 0 до $5 \mathrm{MHz}$,

$$
R f_{i n d, t}=R_{i n d, t} \otimes h_{p r}(i)+N_{i n d, t},
$$

где $h_{p r}(i)$ - импульсная функция электрического усилителя, $N_{i n d, t}$ - оставшиеся физические (от колебания

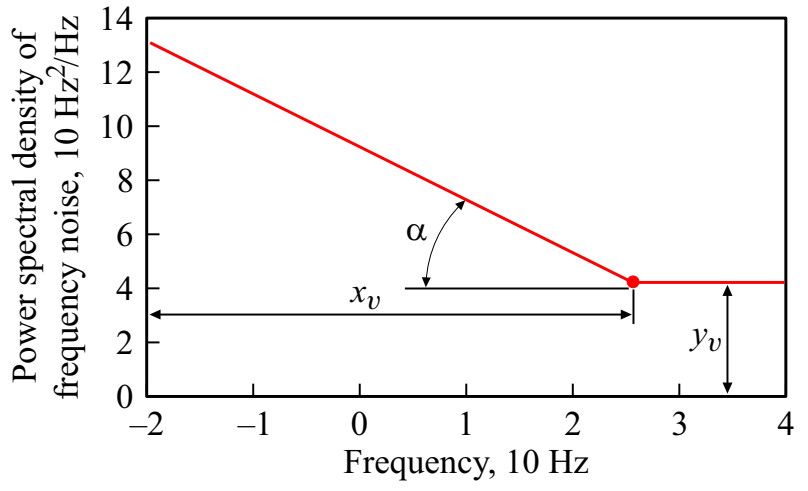

Рис. 2. Схема определения параметров для моделирования лазера.

центров рассеяния), оптические (от предусилителя) и электрические шумы системы.

При отсутствии внешнего сигнального воздействия на изменения влияют два основных фактора:

1. флуктуации центральной длины волны лазера $v_{t}$ во времени,

2. колебания центров рассеяния под действием температурных и случайных внешних механических воздействий на сенсорное волокно.

В большинстве случаев изменение сигнала вызвано именно первой причиной, если только волокно не уложено в зоне с сильными постоянными шумовыми воздействиями.

При производстве лазерных источников наиболее распространенной характеристикой стабильности центральной длины волны является график спектральной плотности мощности флуктуаций частоты $S_{v}$. Ее будем задавать в диапазоне от $1 / T=0.01 \mathrm{~Hz}$ (для задания на отрезке длительностью $T=100 \mathrm{~s}$, чтобы убрать краевые эффекты) до частоты посылки рефлектограмм $v_{p}=1 \mathrm{kHz}$ (частота получения рефлектограмм).

Обычно производитель дает вид огибающей графика $\hat{S}_{v}$, которую для получения реалистичной временной зависимости необходимо домножить на нормированную спектральную плотность случайного равномерного сигнала:

$$
S_{v}=\hat{S}_{v}|F\{\operatorname{rand}(1)\}|^{2} \frac{1}{v_{p}^{2} T},
$$

где $F\{\ldots\}$ - преобразование Фурье.

Затем график зависимости частоты от времени может быть получен с помощью преобразования

$$
v_{t}=v_{0}+\left|F^{-1}\left\{\sqrt{S_{v} v_{p}^{2} T}\right\}\right|,
$$

где $v_{0}$ - частота лазера в начальный момент времени, $F^{-1}\{\ldots\}-$ обратное преобразование Фурье.

Общий вид огибающей удобно задавать в виде графика, представленного на рис. 2.

Он состоит из линейно убывающей и постоянной частей. В таком виде его удобно задавать с помощью трех 
параметров: тангенса угла наклона убывающей части, а также частоты и значения спектральной плотности в точке перегиба.

Данная модель позволяет получать симулированный сигнал с фазочувствительного рефлектометра, использующего определенный лазер с заданной характеристикой, но не демонстрирующий внешних воздействий. Для их создания необходимо задать деформации сенсорного волокна $\Delta L_{n, t}$ под действием внешнего вибрационного сигнала. Процесс передачи механических колебаний от окружающей среды к волоконному кабелю является сложным и многопараметрическим, его протекание будет зависеть от множества характеристик. Например, для грунта это будут плотность, влажность, вязкость, температура, гранулированность, смачивание. Также в каждом случае по-разному будет передавать воздействие оболочка волокна (армирование кабеля, при наличии). Из-за большой сложности этот вопрос не рассматривается подробно, и процесс передачи внешних воздействий интерпретируется следующим алгоритмом:

1. воздействие, происходящее в определенной точке, вызывает одиночное импульсное смещение центров рассеяния на величину $\Delta L_{\text {max }}$;

2. после получения начальной деформации центры рассеяния начинают колебаться с периодом $P(s)$, определяемым свойствами окружающей среды и волокна $s$, затухая с постоянной по времени $r_{t}(s)$;

3. колебания также распространяются от места первоначального воздействия со скоростью $v(s)$, и затухая с постоянной по расстоянию $r_{n}(s)$.

Тогда воздействие, произошедшее в момент времени $t_{c}$ на участке $n_{c}$ сенсора, можно задать выражением

$$
\begin{aligned}
& \Delta L_{n, t}\left(\Delta L_{\max }, s, n_{c}, t_{c}\right)= \\
& =\left\{\begin{array}{l}
\Delta L_{\max } \exp \left(-\frac{\left|n-n_{c}\right|}{\tau_{n}(s)}\right) \exp \left(-\frac{t-t_{c}}{\tau_{t}(s)}\right) \\
\quad \times \sin \left(\frac{t-t_{c}-\frac{d_{3}\left|n-n_{c}\right|}{v(s)}}{P(s)}\right) \text { if } t \geq t_{c}, \\
0 \quad \text { otherwise }
\end{array}\right.
\end{aligned}
$$

Данная величина является добавочным слагаемым к положению центров рассеяния $L_{n}$ в выражении (4). Задавая набор воздействий в соответствии с выражением (12), мы можем получать симулированные сигналы с сенсорной системы с внешними воздействиями.

\section{Экспериментальная часть}

Для проверки предложенной модели и ее показаний мы сравниваем численные результаты с экспериментами для трех лазерных источников. Для реализации такого эксперимента схема фазочувствительного рефлектометра была дополнена узлом, обеспечивающим параллельное с регистрацией сигнала измерение длины волны

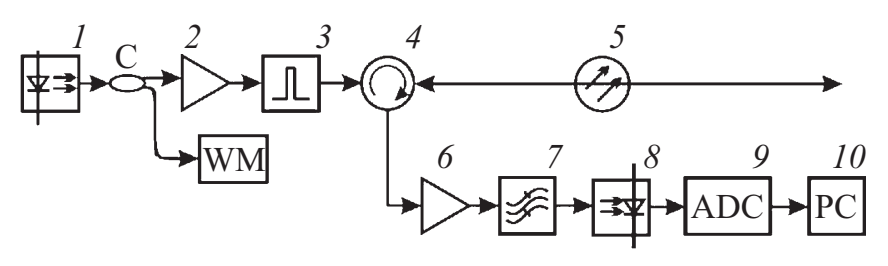

Pис. 3. Дополненная схема фазочувствительного рефлектометра.

Сравнение СКО для экспериментальных и смоделированных данных

\begin{tabular}{c|c|c|c|c}
\hline \multirow{2}{*}{ Лазер } & \multicolumn{2}{|c|}{ СКО длинного участка } & \multicolumn{2}{c}{ СКО короткого участка } \\
\cline { 2 - 5 } & эксперимент & модель & эксперимент & модель \\
\hline OE & 0.1336 & 0.1188 & 0.1226 & 0.1175 \\
DL & 0.1150 & 0.1043 & 0.0297 & 0.0313 \\
RIO & 0.0431 & 0.0424 & 0.0345 & 0.0360
\end{tabular}

источника. Для этого часть излучения лазера через разветвитель $C$ отводится на измеритель длины волны WM.

В такой схеме (рис. 3) становится возможным измерение одновременно и сигнала рефлектометра, представленного на рис. 4, и спектральной плотности мощности флуктуаций частоты каждого источника, представленной на рис. 5. На рис. 4 для наглядности также приведено изменение частоты лазера на отрезке времени, в котором был зарегистрирован сигнал рефлектометра.

На основе измеренных спектральных плотностей источников были смоделированы сигналы рефлектометра с этими источниками на основании созданной модели (без воздействия). Сравнить результаты моделирования пространственно-временного распределения поля $I(l, t)$ и экспериментальных данных можно визуально на рис. 6 , a также по значениям СКО на коротких $(50 \mathrm{~ms})$ и длинных $(1000 \mathrm{~ms})$ участках, приведенных в таблице. Видно, что получаемые результаты находятся в хорошем соответствии друг с другом, что говорит о корректности разработанной модели.

\section{Расчетная часть}

Для определения количественных характеристик лазерных источников, обеспечивающих работоспособность фазочувствительного рефлектометра, смоделируем сигнал от системы с лазерами, имеющими различные характеристики.

1. График состоит только из линейной убывающей части и равномерной шумовой подложки, для моделирования задается положение точки перегиба $\left(x_{v}, y_{v}\right)$.

2. Убывающая линейно часть графика будет иметь наклон $\alpha$ от -20 до $-30 \mathrm{~dB} /$ order.

3. Для определения соотношения сигнал/шум использовался следующий алгоритм. Синусоидальный сигнал 

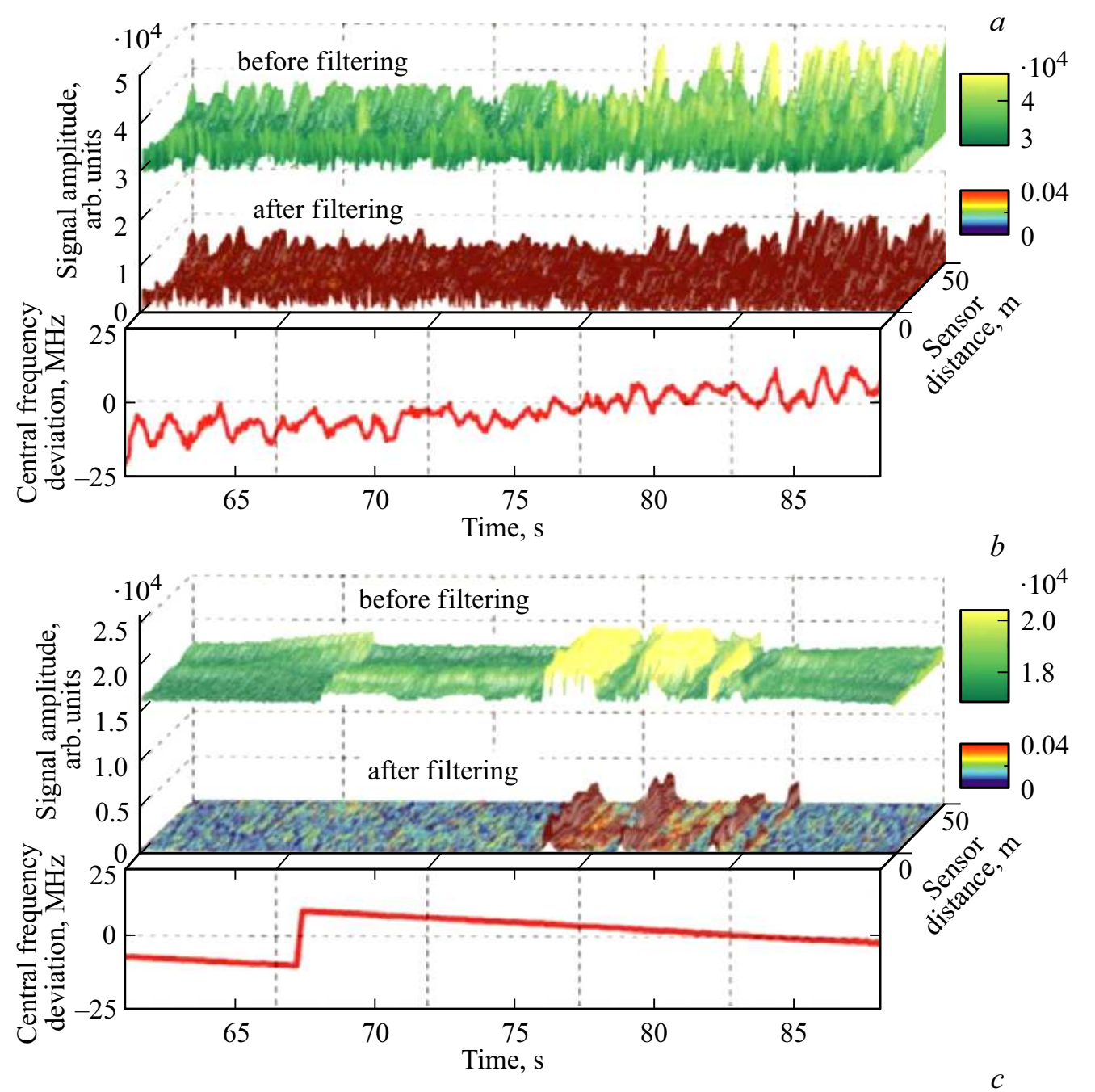

$\cdot 10^{4}$

2.0

$-1.8$

$c$

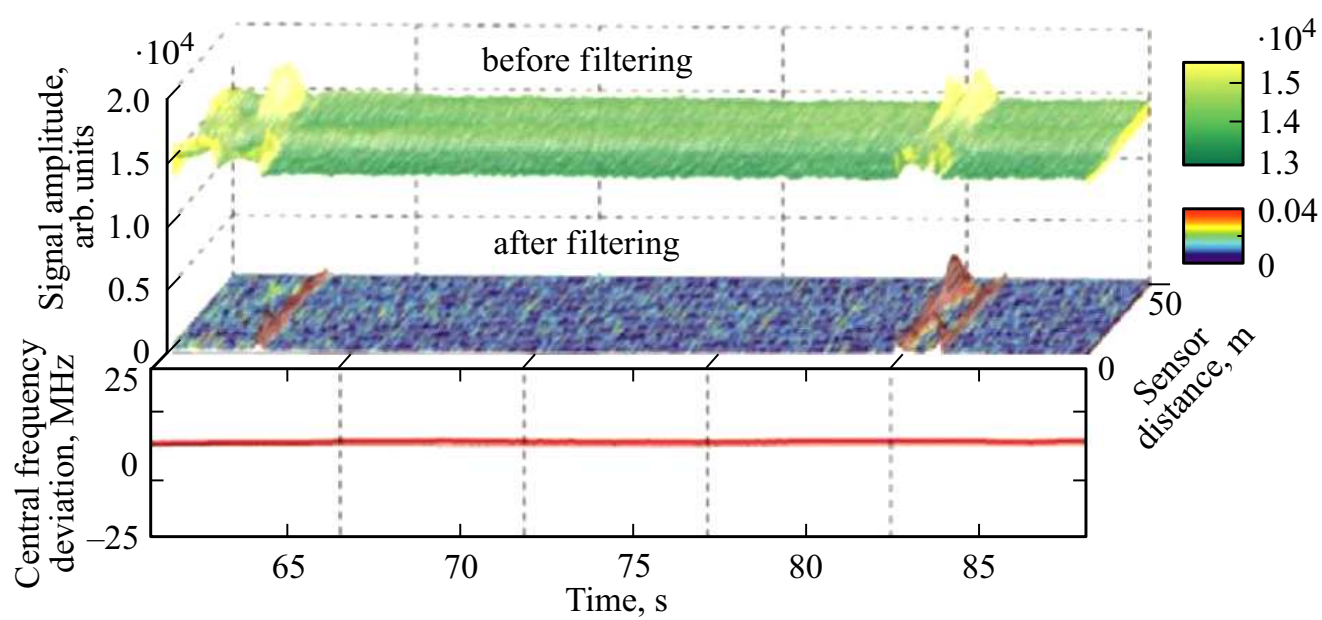

Рис. 4. Образцы сигналов для $(a)$ OE Waves, $(b)$ Dense Light и $(c)$ RIO, записанные одновременно с регистрацией длины волны источника. Также приведены эти сигналы, пропущенные через полосовой фильтр от 8 до $40 \mathrm{~Hz}$.

генерировался на первых $60 \mathrm{~m}$ сенсора. Так как для заданной длины импульса генерируемая интенсивность одного отсчета находилась в диапазоне от 0 до 40 arb. units, то среди первых метров сенсора выбирался участок со средней интенсивностью, наиболее приближенной к значению 20 arb. units. Данная величина позволяет оценивать сигнал на участке со средней чувствительностью, избегая участков слишком низкой и слишком высокой чувствительности. Для этого участка вычисляется СКО сигнала от времени, нормированное на среднее значение 

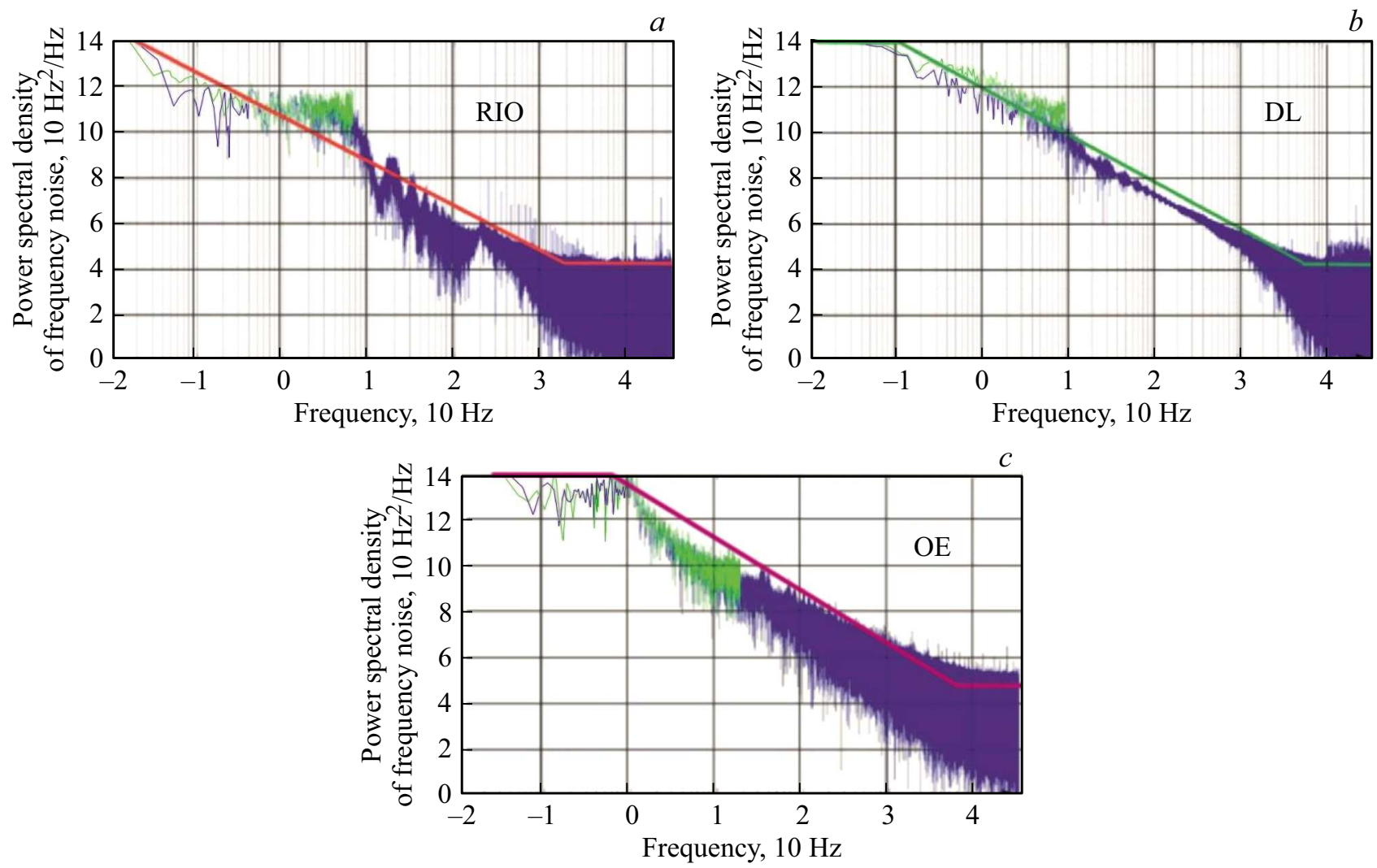

Pис. 5. Графики спектральной плотности флуктуаций частоты лазеров: (a) RIO, $(b)$ DL и $(c)$ OE Waves.
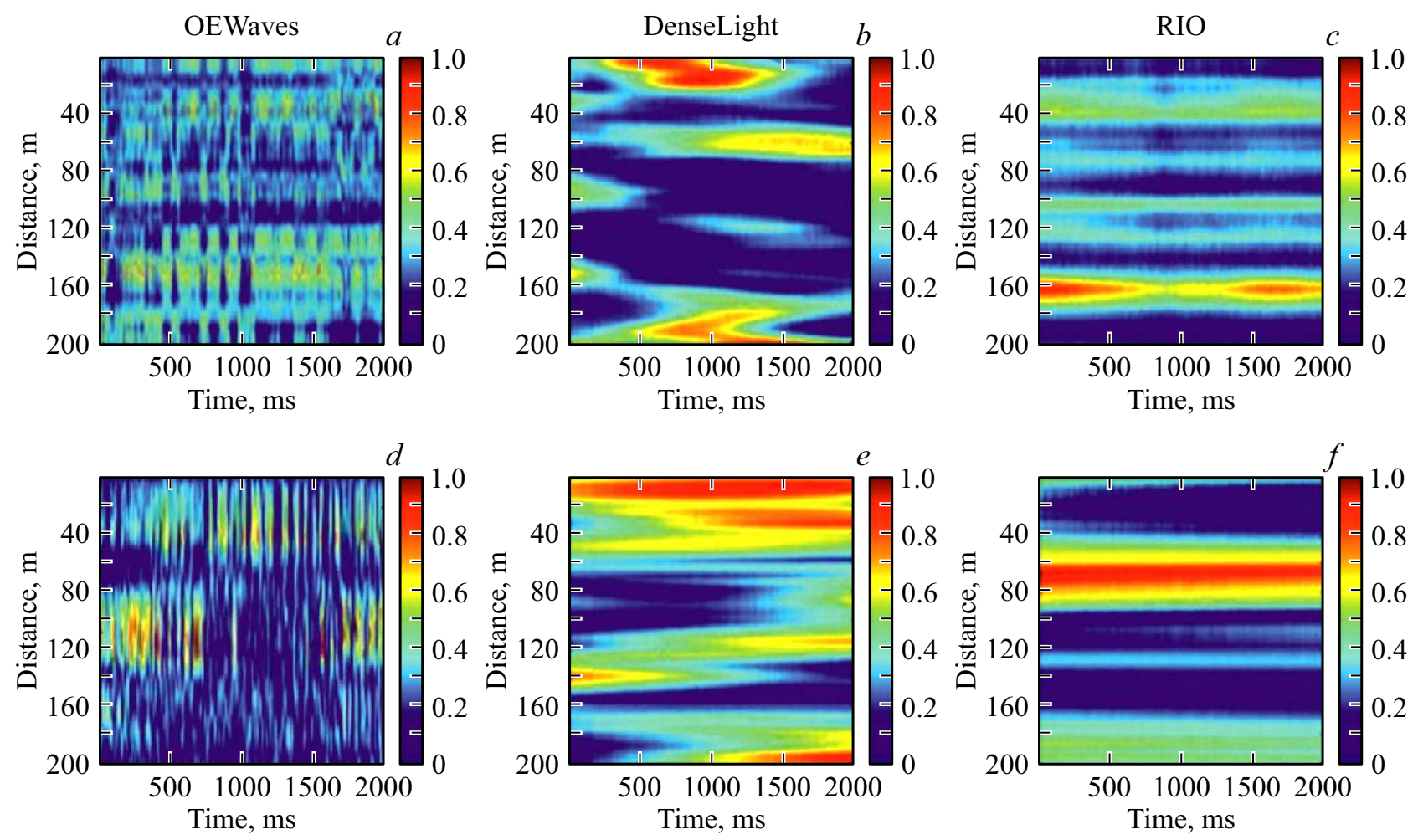

Рис. 6. Графики сигналов фазочувствительного рефлектометра: $a, b, c-$ экспериментальные данные, $d, e, f-$ данные, полученные численным моделированием. 


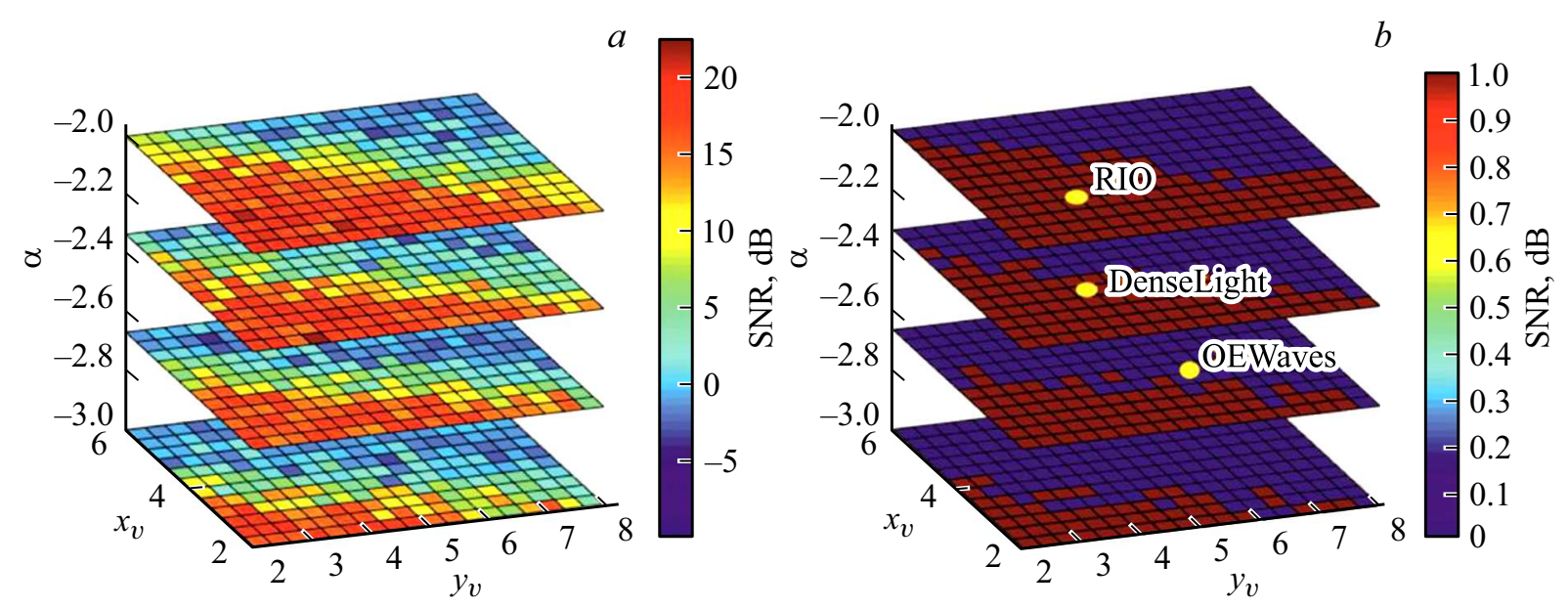

Pис. 7. График отношения сигнал/шум $(a)$ и график области лазеров $(b)$, обеспечивающих $\mathrm{SNR}>10$.

на этом участке за $N$ рефлектограм,

$$
\begin{gathered}
S T D_{\text {sig }}=\frac{1}{J_{\text {sig }}} \sqrt{\frac{\Sigma_{1}^{N}\left(J_{i, j 20}-\bar{J}\right)^{2}}{n}}, \\
\overline{J_{\text {sig }}}=\Sigma_{1}^{N} J_{i, j 20 .}
\end{gathered}
$$

Для участка, на котором воздействие не генерировалось, определялось нормированное СКО для участков с минимальным и максимальным средними значениями, а за итоговое значение принималось их среднее арифметическое. Полученное значение считается величиной шума в данном моделировании,

$$
\begin{aligned}
S T D_{\text {noise }}= & \frac{1}{2 \bar{J}} \sqrt{\frac{\sum_{1}^{N}\left(J_{i, j \min }-\overline{J_{\min }}\right)^{2}}{n}} \\
& +\frac{1}{2 \bar{J}} \sqrt{\frac{\sum_{1}^{N}\left(J_{i, j \max }-\overline{J_{\max }}\right)^{2}}{n}}, \\
\overline{J_{\min , \max }} & =\sum_{1}^{N} J_{i, j \min , \max } .
\end{aligned}
$$

Таким образом, уровень сигнал/шум определяется их отношением:

$$
S N R=\frac{S T D_{\text {sig }}}{S T D_{\text {noise }}} .
$$

Результаты моделирования в виде цветовой карты представлены на рис. 7, $a$. Область источников, обеспечивающая в сигнале системы отношение сигнал/шум более 10, выделена цветом на рис. 7, $b$.

На основании полученных данных видно, что из трех использованных лазеров в эксперименте два дают возможность получения качественного сигнала, а один нет. Эти результаты совпадают с визуальным отображением на рис. 4. На нем представлены сигналы после фильтрации в полосе от 8 до $40 \mathrm{~Hz}$. При этом на первых двух графиках в сигнале становятся видны сигналы удары по сенсору, в то время как на третьем остается только постоянно флуктуирующий сигнал, который не позволяет выделить события.

Исходя из графика на рис. 7, $b$ можно сказать, что для получения сигнала высокого качества в рефлектометре необходимо применять лазер, у которого спектральная плотность мощности флуктуаций частоты не превышает $10^{3} \mathrm{~Hz}^{2} / \mathrm{Hz}$ на частоте $1 \mathrm{kHz}$. Однако для более точного определения отношения сигнал/шум для тестируемого лазерного источника лучшим решением будет подстановка его спектральной плотности флуктуаций частоты в модель и последующий расчет SNR.

\section{Заключение}

Разработана математическая модель формирования сигнала фазочувствительного рефлектометра, учитывающая флуктуации частоты лазерного источника, корректность которой подтверждена сходимостью с экспериментальными данными. С помощью серии численных расчетов на основе данной модели предложены количественные критерии к источникам, обеспечивающим высокое качество сигнала фазочувствительного рефлектометра.

\section{Финансирование работы}

Работа А.А. Жирнова и К.В. Степанова по теоретическому и экспериментальному исследованию стабильности лазерного источника выполнена при финансовой поддержке РФФИ в рамках научного проекта № 18-3200688. Работа А.К. Федорова выполнена при финансовой поддержке РФФИ в рамках научного проекта № 18-3720033.

\section{Конфликт интересов}

Авторы заявляют, что у них нет конфликта интересов. 


\section{Список литературы}

[1] Bao X., Chen L. // Sensors. 2012. T. 12. N 7. C. 8601-8639.

[2] Taylor H.F., Lee C.E. US patent 5, 194847, 1993.

[3] Park J., Lee W., Taylor H.F. // Optical and Fiber Optic Sensor Systems. International Society for Optics and Photonics. 1998. V. 3555. P. 49-57.

[4] Choi K.N., Taylor H.F. // IEEE Photonics Technology Lett. 2003. V. 15. N 3. P. 386-388.

[5] Juarez J.C., Maier E.W., Choi K.N., Taylor N.F. // J. Lightwave Technology. 2005. V. 23. N 6. P. 2081-2087.

[6] Rao Y.J., Luo J., Ran Z.L., Yue J.F., Luo X.D., Zhou Z. // 20th International Conference on Optical Fibre Sensors. International Society for Optics and Photonics. 2009. V. 7503. P. 750310.

[7] Lu Y., Zhu T., Chen L., Bao X. // J. Lightwave Technology. 2010. V. 28. N 22. P. 3243-3249.

[8] Masoudi A., Newson T.P. // Optics Express. 2017. V. 25. N 25. P. 32021-32040.

[9] Martins H.F., Martin-Lopez S., Corredera P., Filograno M.L., Frazao O., Gonzalez-Herraez M. // J. Lightwave Technology. 2013. V. 31. N 23. P. 3631-3637.

[10] Nesterov E.T., Zhirnov A.A., Stepanov K.V., Pnev A.B., Karasik V.E., Tezadov Ya.A., Kondrashin E.V., Ushakov A.B. // J. Physics: Conference Series. IOP Publishing. 2015. V. 584. N 1. P. 012028.

[11] Alekseev A.E., Vdovenko V.S., Gorshkov B.G., Potapov V.T., Simikin D.E. // Laser Physics. 2016. V. 26. N 3. P. 035101.

[12] Nikitin S.P., Ulanovskiy P.I., Kuzmenkov A.I., Nanii O.E., Treshchikov V.N. // Laser Physics. 2016. V. 26. N 10. P. 105106.

[13] Alekseev A.E., Vdovenko V.S., Gorshkov B.G., Potapov V.T., Simikin D.E. // Laser Physics. 2015. V. 25. N 6. P. 065101.

[14] Alekseev A.E., Tezadov Y.A., Potapov V.T. // J. Commun. Technology and Electronics. 2011. V. 56. N 12. P. 1490-1498.

[15] Gabai H., Eyal A. // Optics Lett. 2016. V. 41. N 24. P. $5648-$ 5651.

[16] Alekseev A.E., Tezadov Y.A., Potapov V.T. // Technical Phys. Lett. 2012. V. 38. N 1. P. 89-92.

[17] Li Q., Zhang C., Li L., Zhong X. // Optik-Intern. J. Light and Electron Optics. 2014. V. 125. N 9. P. 2099-2103.

[18] Liokumovich L.B., Ushakov N.A., Kotov O.I., Bisyarin M.A., Hartog A.H. // J. Lightwave Technology. 2015. V. 33. N 17. P. 3660-3671.

[19] Zhong X., Zhang C., Li L., Liang S., Li Q., Lii Q., Ding X., Cao Q. // Appl. Optics. 2014. V. 53. N 21. P. 4645-4650.

[20] Pnev A.B., Zhirnov A.A., Stepanov K.V., Nesterov E.T., Shelestov D.A., Karasik V.E. // J. Physics: Conference Series. IOP Publishing. 2015. V. 584. N 1. P. 012016.

[21] Goodman J.W. Statistical Optics. NY:. Wiley-Interscience, 1985. 